\title{
Tuberculous Osteomyelitis of the Foot: A Case Report
}

\author{
Zahra Abdi ${ }^{1}$; Fereshte Ghiasvand ${ }^{1,}$; Seyed Ali Dehghan Manshadi ${ }^{1}$ \\ ${ }^{1}$ Department of Infectious Diseases and Tropical Medicine, Tehran University of Medical Sciences, Tehran, IR Iran \\ ${ }^{*}$ Corresponding author: Fereshte Ghiasvand, Department of Infectious Diseases and Tropical Medicine, Tehran University of Medical Sciences, Tehran, IR Iran. Tel: +98-2166581598, \\ Fax: +98-2166581598, E-mail: ghiasvand_62@yahoo.com
}

Received: December 15, 2013; Accepted: December 30, 2013

\begin{abstract}
Introduction: Tuberculosis of the foot is an unusual type of skeletal tuberculosis.
Case Presentation: We report on an eighty-six-year-old woman, who presented pain and purulent discharge from the right foot for 10 months. The final diagnosis was confirmed with a culture of Mycobacterium tuberculosis.

Discussion: Diagnosis of this form of tuberculosis is established with some delay due to the uncommon site of involvement, lack of awareness about the condition and its ability to mimic other disorders. Diagnosis and appropriate treatment can often be delayed, which may lead to significant consequences for the patient. Our case was treated with anti-tuberculin medications for nine months and conservative management of the bony lesions resulting complete resolution of symptoms.
\end{abstract}

Keywords:Tuberculosis; Osteomyelitis; Foot

\section{Introduction}

Tuberculosis still remains a major problem in the developing world. Bones and joints are involved in 1\% to 3\% of all cases and about $10 \%$ of skeletal tuberculosis affects the foot (1). We reported a case of TB osteomyelitis of foot which presented as severe purulent wound.

\section{Case Presentation}

An eighty-six-year-old woman, who was a known case of major depression disorder and dementia, was admitted to the emergency department of our hospital due to loss of consciousness. Her problem had begun one week ago with coughs, sputum, fever, chills, progressive dyspnea and loss of consciousness. Regarding her past medical history, she had pain and swelling of the dorsal aspect of her right foot since 10 months ago for which drainage was performed with diagnosis of subcutaneous abscess but she had not improved and had became bed ridden since then. On physical examination, she was confused with a temperature of $38^{\circ} \mathrm{C}$, blood pressure of $116 / 80 \mathrm{~mm}$ $\mathrm{Hg}$, pulse rate of 90 beats per minute and respiratory rate of 26 per minute. She had bilateral diffuse crackles on pulmonary examination. She also had a wound with 2 cm diameter and thick purulent discharge from her right foot without significant erythema. Other physical examinations were normal.

Regarding hematological laboratory tests, she had leukocytosis with white blood cell (WBC) count of $26000 /$ $\mu \mathrm{L}$ and platelet count of $370000 / \mu \mathrm{L}$ while her hemoglo- bin level was 10/5 Mg/dL. Eererythrocyte sedimentation rate (ESR) was 81 and C-reactive protein (CRP) was 79. Her blood and urine cultures and culture from wound discharge were all negative. PPD was $4 \mathrm{~mm}$. The chest radiography and computerized tomography (CT) scan showed bilateral patchy infiltrations. She was treated with antibiotics (meropenem $1 \mathrm{~g}$ three times a day and vancomycin $1 \mathrm{~g}$ two times a day ) for pneumonia and after a few days her respiratory symptoms and fever subsided and leukocyte count dropped to $8600 / \mu \mathrm{L}$. The patient was well, yet her wound's discharge increased. She had severe pain and copious purulent discharge from her foot. Gram stain smear and culture of the wound discharge for routine pyogenic bacteria were negative and her foot plain radiograph showed no significant abnormality, yet ultrasonography reported collection with internal echo in subcutaneous tissue with a fistula to the skin surface. We sent a sample of the wound discharge for Ziehl-Neelsen staining and culture for tuberculosis. Acid-fast bacilli were found on microscopic examination. Foot magnetic resonance imaging (MRI) reported osteomyelitis of calcaneus, cuboid, anteromedial part of talus, navicular bone and cuneiforms (Figure 1). Anti-tuberculosis treatment was started with the standard four-drug regimen (iso niazid, rifampin, pyrazinamide and ethambutol). After two months of treatment, wound discharge stopped, fistula closed and dorsal foot pain decreased. The result of wound culture for mycobacterium tuberculosis was also positive after two months. 
Figure 1. Abnormal Bone Marrow Signal and Bone Destruction in Calcaneous and Cuboid

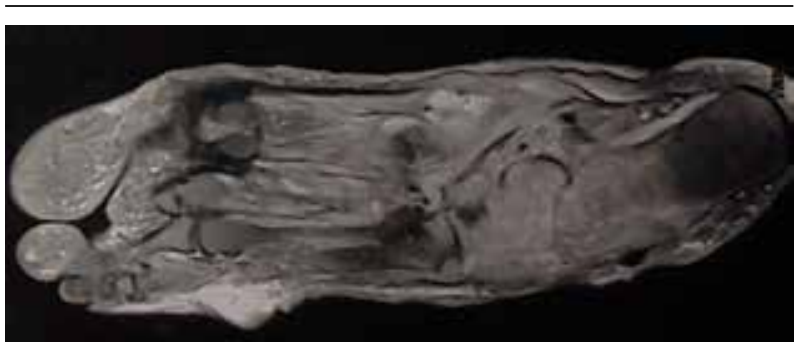

Osteomyelitis is noted in anteromedial part of talus, navicular bone and cuneiforms.

\section{Discussion}

Prevalence of tuberculosis is increasing in developed countries, but tuberculous osteomyelitis of the foot remains an uncommon complication and may become an important problem (2-5). Despite Skeletal tuberculosis is extremely rare, but the most commonly affected sites are the spine and large joints (2) and involvement of foot accounts for less than $10 \%$ of osteoarticular tuberculosis (6). This condition results in tuberculous misdiagnosis, or the true nature of the lesion is identified late in the diagnostic process (6). Also, because of non-specific symptoms and unhelpful hematological investigations, diagnosis is difficult while variable clinical and radiological features can mimic phylogenic osteomyelitis, bone tumor or other inflammatory and neoplastic processes of the synovium (3). Identifying acid-fast mycobacteria from skeletal tuberculosis is difficult (1). In our patient, wound smear for acid-fast bacilli was positive. Thus, tuberculosis should be con- sidered in the differential diagnosis when a patient presents localized, painful swelling and a persistent draining sinus of the foot and ankle. Taking a biopsy of the site of suspected infection is recommended because an early diagnosis is the key to successful treatment (3). In our case, the patient was treated with antituberculin medications for 9 months with a complete resolution of symptoms. After antituberculous therapy the wound healed completely.

\section{Authors' Contributions}

All authors contributed equally to this work.

\section{Funding/Support}

The authors declare that there was no conflict of interests.

\section{Financial Disclosure}

This study did not receive any financial support.

\section{References}

1. Mittal R, Gupta V, Rastogi S. Tuberculosis of the foot. Bone Joint Surg Br.1999;81(6):997-1000.

2. Kerens B, Gans EH, Pilot P, van der Tempel H, Verburg AD. [Atypical tuberculous osteomyelitis of the humeral shaft caused by Mycobacterium avium]. Ned Tijdschr Geneeskd. 2006;150(31):1728-31.

3. Choi WJ, Han SH, Joo JH, Kim BS, Lee JW. Diagnostic dilemma of tuberculosis in the foot and ankle. Foot Ankle Int. 2008;29(7):711-5.

4. Brew CJ, Rao V, Shanker J. Tuberculosis infection of the talonavicular joint. Foot (Edinb). 2010;20(4):146-8.

5. Ferguson KB, Jones CE, Thomson AG, Moir JS. A rare case of tuberculosis of the midfoot. Foot Ankle Spec. 2012;5(5):327-9.

6. Bozkurt M, Dogan M, Sesen H, Turanli S, Basbozkurt M. Isolated medial cuneiform tuberculosis: a case report. J Foot Ankle Surg. 2005;44(1):60-3. 